\title{
Case Report: Complete Clinical Response of a Young Patient with a BRCA 2 Positive Metastatic Breast Cancer
}

\author{
Grazia Artioli $^{1 *}$, Giuseppe Azzarello ${ }^{1}$, Fabrizio Meggiolaro ${ }^{2}$, Jacopo Wabersich ${ }^{3}$, Lucia Borgato ${ }^{1}$, \\ Mario Bari ${ }^{1}$ \\ ${ }^{1}$ Department of Medical Science, Oncology-Haematology, Mirano, Italy \\ ${ }^{2}$ Department of Surgery, Mirano, Italy \\ ${ }^{3}$ Department of Obstetrics and Gynecology, Mirano, Italy \\ Email: *grazia.artioli@yahoo.it
}

Received March 23, 2013; revised April 26, 2013; accepted May 3, 2013

Copyright (C) 2013 Grazia Artioli et al. This is an open access article distributed under the Creative Commons Attribution License, which permits unrestricted use, distribution, and reproduction in any medium, provided the original work is properly cited.

\begin{abstract}
Background: Metastatic breast cancer in BRCA 2 patients is lacking of target therapy studies. Case: We present a case of a 34-year-old woman affected by a recurrent metastatic breast cancer after 5 years treated with weekly nab-paclitaxel and three weekly Cisplatin obtaining a radiological complete response with 6 cycles. Conclusion: It is important to emphasize that patients with a molecular disorder who selected for "target" chemotherapy can obtain optimal response rate. Exploiting the mechanism of action of DNA damage in our favor, using cisplatin for example and basing on literature show the efficacy of using taxane in BRCA 2 patients, obtaining a very high response rate.
\end{abstract}

Keywords: BRCA 2; Breast Cancer; DNA; Cisplatin; Taxane

\section{Introduction}

The estimated penetrance of ovarian cancer (OC) in BRCA positive women is respectively of $35 \%-60 \%$ for BRCA 1 and $10 \%-27 \%$ for BRCA 2 in 70 -year-old patients. For breast cancer, the penetrance is $87 \%$ and $84 \%$ for BRCA 1 and BRCA 2, respectively in 70 year old patients [1-3]. Platinum drugs are known to induce greater cytotoxicity against BRCA 1 and BRCA 2 cell lines, presumably because when they form a covalent bond with DNA strands, repair cannot proceed by homologous recombination. Preclinical studies suggest that lack of functioning BRCA 1 and/or BRCA 2 protein may result in differential treatment response to several chemotherapeutic drugs. Several studies have reported the sensitivity to apoptosis in BRCA 1 and/or BRCA 2 deficient breast cancer cells treated with Poly ADP inhibitors, cisplatin, mitoxantrone, etoposide and anthracyclines. Nowadays consensus regarding the most effective chemotherapy regimes in BRCA breast cancer mutation carriers is still lacking.

\section{Case Report}

In 2007, a 29-year-old woman was referred to our hospital for a diagnosis of breast cancer. In April 2007, she

${ }^{*}$ Corresponding author. underwent left mastectomy and lymphadenectomy for a multifocal ductal carcinoma, moderate grade, maximum diameter of $3 \mathrm{~cm}$, endolimphatic carcinosis, 4 over 13 positive lymph-nodes. Positive estrogen receptor in $90 \%$ (clone 1 D5) and progesterone in 40\% (clone PgR 636) p 53 0\%, ki 67 5\%, c-erbB 2 0\%. Stadiation exams were all negative for metastases. Stage pT2 N2a M0. She was treated with 3 cycles of FEC (5-fluorouracil, epirubicin and cyclophosfamide) followed by 3 more cycles of docetaxel.

The first administration of docetaxel was complicated by a grade 3 hypersensitivity reaction with dyspnea and tingling resistant to corticosteroids treatment. For this reason, docetaxel was suspended and the patient received 3 more cycles of FEC followed by adjuvant endocrine therapy with LH-RH analogous (triptorelin) and tamoxifen $20 \mathrm{mg} / \mathrm{die}$.

In February 2009, the patient referred an endometrial polyp with mucous hemorrhagic areas and therefore the oncologist suspended the use of tamoxifen, substituting it with an aromatase inhibitor.

In April 2010, the patient underwent breast implant replacement.

In October 2010, after performing a genetic test during a genetic counseling, the patient was found to be BRCA 2 gene (mut 6872 del ACTC $\rightarrow$ ter 2227) carrier. 
She continued hormonal therapy and follow up, integrating it with breast MRI and with mammography and transvaginal ultrasound every 6 months plus CA 125 dosage.

In October 2011, the patient referred to her oncologist that she did not feel well, in particular lamenting a shoulder pain. Her performance status was 2 and the blood tests showed a rise of CEA and CA 15.3.

Due the alteration of serum markers, the oncologist decided to perform a total body FDG PET-CT scan which revealed the presence of a metastatic disease diffusion with liver, bone and lymph node metastases, as shown in Figure 1(a).

The patient underwent liver biopsy, which showed a recurrence of breast ductal carcinoma, G2, with positive estrogen receptor $90 \%$ (Clone ER-SP1), progestinics $4 \%$, (Clone PgR 1E2) c-erbB2 $1+$ and p53 10\%. On December, 2nd, 2011, after signing the informed consent, the patient started chemotherapy with cisplatin $75 \mathrm{mg} / \mathrm{m}^{2}$ day 1 and protein-bound pacitaxel $90 \mathrm{mg} / \mathrm{m}^{2}$ days $1,8,15 \mathrm{q} 3$ weeks, for a total of 6 cycles, plus zoledronic acid every 28 days. During the cycles of therapy, a gradual decline of tumor-markers CEA and CA 15.3 were evident, together with physical recovery: the patient was able to return to work in a few months and lead an almost normal life, her PS 0.

After 6 cycles of therapy, the patient underwent a new PET CT scan evaluation, as shown in Figure 1(b), which showed a complete clinical response. The oncologists decided a treatment with LH RH analogous and to start tamoxifene $20 \mathrm{mg} /$ die monitoring endometrial thickness and monthly zoledronic acid.

After five months of remission, because of increased tumor markers CEA without any symptoms, patient underwent a chest and abdomen CT scan which revealed diffused bone dysostoses. After other two months, a further increase of tumor markers was observed and therefore she underwent a PET-CT scan, which showed a 1 $\mathrm{cm}$ paratracheal lymph node and diffused bone metastases in the still asymptomatic patient. In the light of asymptomatic conditions, previous leuco-neutropenia and absence of visceral disease, it was decided to start with a new hormonal regimen, LH-RH analogous with fulvestrant.

\section{Discussion}

This case report aims to highlight the problems linked to the presence of a genetic mutation and the possibility of directing chemotherapy by exploiting the mechanism of action of the mutation.

The pathways of DNA damage response and DNArepair are affected by different kind of genetic mutations in BRCA 1 and BRCA 2 cancer. In those cells, the DNA repair is not able to work and damaged cells, by chemo-

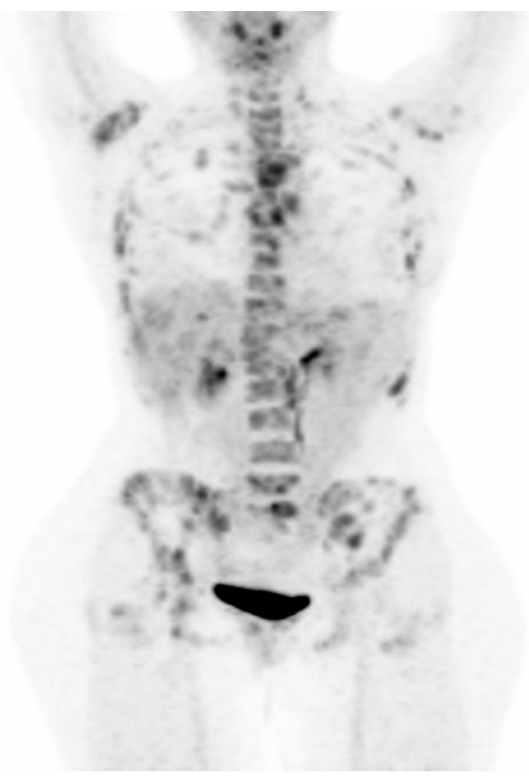

(a)

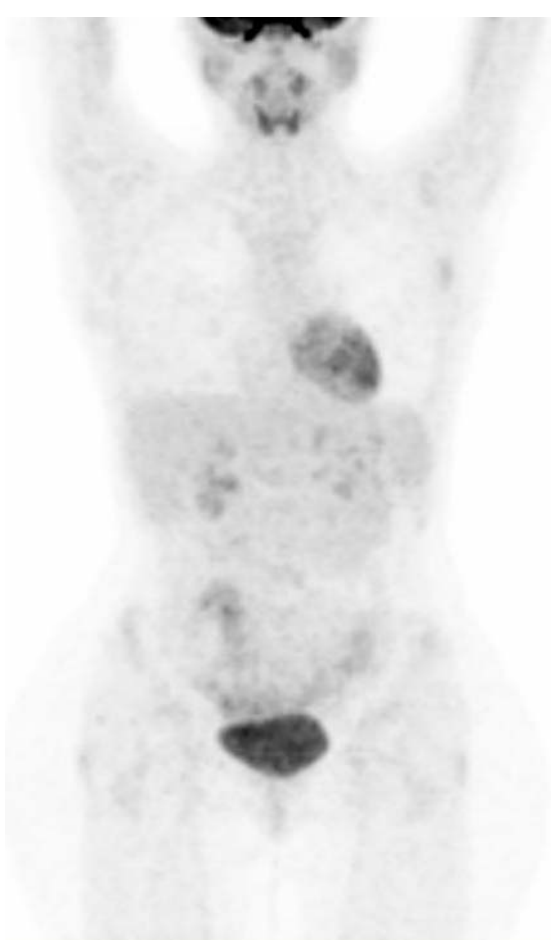

(b)

Figure 1. PET-CT evaluation before (a) and after (b) treatment.

therapy, apoptose. Using this mechanism in order to potentiate the effect of chemotherapeutic agents as cisplatin, alkylating agents or PARP inhibitors is well known, yet literature in this field is lacking.

Protocols are underway to evaluate new drugs called poly-(ADP-ribose) polymerase inhibitors, specifically designed to exploit the sensitivity of BRCA 1 associated cancer to DNA damage. Olaparib, a novel, orally active 
poly (ADP-ribose) polymerase (PARP) inhibitor, induced synthetic lethality in BRCA-deficient cells and the prevalence of BRCA 1 inactivation in triple negative breast cancer has rationalized clinical trials of poly (adenosine diphosphate ribose) polymerase (PARP) inhibitors. Unfortunately PARP inhibitors failed in demonstrating an advantage in terms of response rate in triple negative breast cancer, but it seems to be promising in ovarian cancer treatment in BRCA mutated women.

For example, the phase 2 study of Gelmon failed to demonstrate an advantage of PARP inhibitor in breast cancer probably because authors wanted to test all triple negative breast cancer without distinguishing between BRCA positive and basal like sporadic cancer. In Tutt's study, instead, olaparib where tested only in BRCA positive breast advanced cancer showing a favourable therapeutic index. In BRCA positive breast cancer mechanism of action of PARP inhibitors is different because the drug is able to exploit the lack of DNA repair genes and the same mechanism could be possibly not well effective in basal like (triple negative) sporadic cancer, where PARP inhibitors cannot display their actions so actively [4-6]. Those results can be justify by Domagala study where he assessed the expression of PARP-1 in different kind of breast cancer, showing a significant difference between the mean nuclear PARP-1 quickscore in BRCA 1-associated versus BRCA 1-non-associated carcinomas in all tumours ( $<$ 0.0001), in the basal-like group ( $p$ 0.0086), triple negative (p 0.0015), and non-basal-like groups ( $\mathrm{p}$ 0.016) but not in the nontriple negative group [7].

From retrospective studies, we know that cisplatin is also a very efficacious drug that exploits DNA damage response, and there is evidence of in vivo therapeutic response to mitomycin C in BRCA 2 mutated pancreatic cancer. A high proportion of women with a BRCA 1 associated breast cancer responded to platinum based chemotherapy and cisplatin alone can give 83\% (10/12) of pathologic response rate in the neoadjuvant setting in locally advanced breast cancer BRCA 1 positive women [8]. Kriege's study showed that BRCA 2 associated breast cancer patients had a higher Response Rate (RR) than sporadic patients using taxane chemotherapy ( $\mathrm{p}$ 0.02), but no differences in PFS were seen. In that study, most of BRCA 2 positive breast cancer patients had hormone receptors (HRS) positive cancer. The same result in terms of RR was not as good as among BRCA 1 mutated patients, because most of BRCA 1 patients had HRS negative cancer. No differences in terms of Response Rate in this group were seen using taxane-based chemotherapy [9]. Few cases are reported about efficacy of DNA damaging drugs in metastatic BRCA breast cancer. Huang F., showed that high dose alkylating agents and platinum-based therapy could obtain a complete pathological response in a metastatic BRCA 2 breast can- cer patient [10].

Based on recent data on protein-bound paclitaxel, we decided to use this drug because it is a new, biologically interactive nanometer-sized albumin-bound taxane particle initially developed to avoid the toxicities associated to polyethylated castor oil. The albumin-bound nanoparticle was designed to preferentially deliver paclitaxel to tumours by biologically interacting with albumin receptors that mediate drug transport; in vitro studies have demonstrated a 4.5-fold increase in paclitaxel transport across endothelial cells for protein-bound paclitaxel compared with standard paclitaxel.

A large international phase III study demonstrated that this drug, a new 130 nanometer albumin-bound paclitaxel particle, was superior to standard paclitaxel for both ORR and TTP in all patients with metastatic breast cancer. Protein-bound paclitaxel dose used in this study (50\% higher than the dose delivered with solvent-based paclitaxel) could be administered safely over 30 minutes without premedication [11]. Nab-paclitaxel enhances tumor targeting through gp 60 and caveolae-mediated endothelial transcytosis and the association with the albumin-binding protein SPARC (secreted protein, acidic and rich in cysteine) in the tumor microenvironment [12].

In recent data, for their clinical and pathological characteristics, BRCA positive breast cancer has proved to be more frequent in premenopausal patients with high rate of positive lymph nodes, high grade of differentiation and negative estrogen receptors (around 80\% in BRCA1 mutated breast cancer and 25\% of BRCA 2 mutated breast cancer) $[13,14]$. Despite those negative prognostic factors, survival rates in BRCA are similar to wild type breast cancer. In BRCA positive ovarian cancer, prognostic factors as grade and stage are worse than sporadic cases (high grade of differentiation and advanced stages at diagnosis), but those characteristics seem to correlate with better survival, probably due to good response to therapy with cisplatin.

Literature now available shows that all data reported in retrospective studies demonstrate the same characteristics and prognostic factors for BRCA positive breast and ovarian cancer and that cisplatin seems to be the most favourable chemotherapy drug in terms of response rate, as well as mitomycin and alkylating agents. New data on the use of taxane in this setting are under discussion.

In this case report, the oncologists tailored the therapy for this patient on her BRCA 2 mutation and, basing on literature data available, the choice was made to use the synergistic actions of cisplatin and protein-bound paclitaxel, which seem to be effective in breast cancer patient and in BRCA 2 mutated patients. It is very interesting to note that, in this patient, the second relapse found with the PET-scan after metastatic first line treatment was not in the liver but only in the bones. This interesting datum 
can be probably associated with the high number of SPARC proteins in the liver. It was hypothesized that because of a SPARC-albumin interaction, tumoral SPARC facilitates the accumulation of albumin in the tumor and increases the effectiveness of albumin-bound paclitaxel (nab-paclitaxel). Desai et al. tested this hypothesis in head and neck cancer correlating the use of nabpaclitaxel and SPARC expression. The final analysis is supportive of the hypothesis that SPARC over expression may correlate with response to nabpaclitaxel [12]. This hypothesis can justify the complete response in the liver in our patient but it should be better analysed in larger prospective trials. Actually, prospective trials focused on correlation of breast and/or ovarian cancer, BRCA status, chemotherapy and outcome appear to be warranted.

\section{REFERENCES}

[1] D. Ford, D. F. Easton and J. Peto, "Estimates of the Gene Frequency of BRCA1 and Its Contribution to Breast and Ovarian Cancer Incidence,” American Journal of Human Genetics, Vol. 57, No. 6, 1995, pp. 1457-1462.

[2] D. Ford, D. F. Easton, M. Stratton, S. Narod, D. Goldgar, P. Devilee, D. T. Bishop, B. Weber, G. Lenoir, J. ChangClaude, H. Sobol, M. D. Teare, J. Struewing, A. Arason, S. Scherneck, J. Peto, T. R. Rebbeck, P. Tonin, S. Neuhausen, R. Barkardottir, J. Eyfjord, H. Lynch, B. A. Ponder, S. A. Gayther and M. Zelada-Hedman, "Genetic Heterogeneity and Penetrance Analysis of the BRCA1 and BRCA2 Genes in Breast Cancer Families. The Breast Cancer Linkage Consortium," American Journal of $\mathrm{Hu}$ man Genetics, Vol. 62, No. 3, 1998, pp. 676-689. doi:10.1086/301749

[3] A. Antoniou, P. D. Pharoah, S. Narod, H. A. Risch, J. E. Eyfjord, J. L. Hopper, N. Loman, H. Olsson, O. Johannsson, A. Borg, B. Pasini, P. Radice, S. Manoukian, D. M. Eccles, N. Tang, E. Olah, H. Anton-Culver, E. Warner, J. Lubinski, J. Gronwald, B. Gorski, H. Tulinius, S. Thorlacius, H. Eerola, H. Nevanlinna, K. Syrjakoski, O. P. Kallioniemi, D. Thompson, C. Evans, J. Peto, F. Lalloo, D. G. Evans and D. F. Easton, "Average Risks of Breast and Ovarian Cancer Associated with BRCA1 or BRCA2 Mutations Detected in Case Series Unselected for Family History: A Combined Analysis of 22 Studies,” American Journal of Human Genetics, Vol. 72, No. 5, 2003, pp. 1117-1130. doi:10.1086/375033

[4] G. von Minckwitz, S. Loibl, A. Maisch and M. Untch, "Lessons from the Neoadjuvant Setting on How Best to Choose Adjuvant Therapies," Breast (Edinburgh, Scotland), Vol. 20, No. S3, 2011, pp. S142-S145.

[5] K. A. Gelmon, M. Tischkowitz, H. Mackay, K. Swenerton, A. Robidoux, K. Tonkin, H. Hirte, D. Huntsman, M. Clemons, B. Gilks, R. Yerushalmi, E. Macpherson, J. Carmichael and A. Oza, "Olaparib in Patients with Recurrent High-Grade Serous or Poorly Differentiated Ovarian Carcinoma or Triple-Negative Breast Cancer: A Phase 2, Multicentre, Open-Label, Non-Randomised Study,” The Lancet Oncology, Vol. 12, No. 9, 2011, pp. 852-861. doi:10.1016/S1470-2045(11)70214-5
[6] B. Arun, S. Bayraktar, D. D. Liu, A. M. Gutierrez Barrera, D. Atchley, L. Pusztai, J. K. Litton, V. Valero, F. MericBernstam, G. N. Hortobagyi and C. Albarracin, "Response to Neoadjuvant Systemic Therapy for Breast Cancer in BRCA Mutation Carriers and Noncarriers: A Single-Institution Experience,” Journal of Clinical Oncology: Official Journal of the American Society of Clinical Oncology, Vol. 9, No. 28, 2011, pp. 3739-3746.

[7] P. Domagala, T. Huzarski, J. Lubinski, K. Gugala and W. Domagala, "PARP-1 Expression in Breast Cancer Including BRCA1-Associated, Triple Negative and Basal-Like Tumors: Possible Implications for PARP-1 Inhibitor Therapy," Breast Cancer Research and TREATMENt, Vol. 127, No. 3, 2011, pp. 861-869. doi:10.1007/s10549-011-1441-2

[8] T. Byrski, J. Gronwald, T. Huzarski, E. Grzybowska, M. Budryk, M. Stawicka, T. Mierzwa, M. Szwiec, R. Wisniowski, M. Siolek, R. Dent, J. Lubinski and S. Narod, "Pathologic Complete Response Rates in Young Women with BRCA1-Positive Breast Cancers after Neoadjuvant Chemotherapy," Journal of Clinical Oncology: Official Journal of the American Society of Clinical Oncology, Vol. 28, No. 3, 2010, pp. 375-379. doi:10.1200/JCO.2008.20.7019

[9] M. Kriege, A. Jager, M. J. Hooning, E. Huijskens, J. Blom, C. H. van Deurzen, M. Bontenbal, J. M. Collee, M. B. Menke-Pluijmers, J. W. Martens and C. Seynaeve, "The Efficacy of Taxane Chemotherapy for Metastatic Breast Cancer in BRCA1 and BRCA2 Mutation Carriers," Cancer, Vol. 118, No. 4, 2012, pp. 899-907. doi:10.1002/cncr.26351

[10] F. Huang, Y. B. Kushner, A. Langleben, W. D. Foulkes and Medscape, "Eleven Years Disease-Free: Role of Chemotherapy in Metastatic BRCA2-Related Breast Cancer," Nature Reviews. Clinical Oncology, Vol. 6, No. 8, 2009, pp. 488-492.

[11] W. J. Gradishar, S. Tjulandin, N. Davidson, H. Shaw, N. Desai, P. Bhar, M. Hawkins and J. O’Shaughnessy, "Phase III Trial of Nanoparticle Albumin-Bound Paclitaxel Compared with Polyethylated Castor Oil-Based Paclitaxel in Women with Breast Cancer," Journal of Clinical Oncology: Official Journal of the American Society of Clinical Oncology, Vol. 23, No. 31, 2005, pp. 7794-7803.

[12] N. P. Desai, V. Trieu, L. Y. Hwang, R. Wu, P. Soon-Shiong and W. J. Gradishar, "Improved Effectiveness of Nanoparticle Albumin-Bound (Nab) Paclitaxel Versus Polysorbate-Based Docetaxel in Multiple Xenografts as a Function of HER2 and SPARC Status," Anti-Cancer Drugs, Vol. 19, No. 9, 2008, pp. 899-909. doi:10.1097/CAD.0b013e32830f9046

[13] A. Veronesi, C. de Giacomi, M. D. Magri, D. Lombardi, M. Zanetti, C. Scuderi, R. Dolcetti, A. Viel, D. Crivellari, E. Bidoli and M. Boiocchi, "Familial Breast Cancer: Characteristics and Outcome of BRCA 1-2 Positive and Negative Cases,” BMC Cancer, Vol. 5, 2005, p. 70.

[14] M. O. Nicoletto, M. Donach, A. De Nicolo, G. Artioli, G. Banna and S. Monfardini, "BRCA-1 and BRCA-2 Mutations as Prognostic Factors in Clinical Practice and Genetic Counselling," Cancer Treatment Reviews, Vol. 27, No. 5, 2001, pp. 295-304. doi:10.1053/ctrv.2001.0233 